\title{
Cognitive assessment of patients with minimal hepatic encephalopathy in Brazil
}

\author{
D. S. Torres • J. Abrantes • C. E. Brandão-Mello
}

Received: 15 November 2012 / Accepted: 4 April 2013 /Published online: 28 April 2013

(C) The Author(s) 2013. This article is published with open access at Springerlink.com

\begin{abstract}
Minimal hepatic encephalopathy is a syndrome caused by liver cirrhosis and accompanied by a broad spectrum of cognitive symptoms. The objective of the present study was to describe the prevalence of minimal hepatic encephalopathy in cirrhotic patients and to compare their cognitive performance with controls using standardized tests. Patients receiving medication or experiencing comorbidities associated with cognitive disorders were excluded. The final cohort was compared with a control-matched group using the Mini-Mental State Examination (MMSE), as well as Simple Drawing, Clock Drawing, Rey Auditory-Verbal Learning Test (RAVLT), Random Letter, Stroop, Trail-Making Test (TMT) A and B, Boston Naming, Category Verbal Fluency, Digit Span, Constructional Praxis, Processing Speed, and Similarities Tests. The results indicated no differences in the prevalence of cognitive complaints spontaneously reported by 29 patients with cirrhosis versus 22 healthy controls. The most affected tests included: MMSE ( $26.3 \pm 2$ vs. $28.1 \pm 1.8$ points; $p=0.004)$, learning ( $35.4 \pm 9$ vs. $41 \pm 9.1$ points; $p=0.041)$, retroactive interference $(0.67 \pm 0.22$ vs. $0.84 \pm 0.16$ points; $p=0.004)$, and recognition ( $8.7 \pm 2.6$ vs. $11.2 \pm 4.1$ points; $p=0.024)$ in RAVLT, TMT-A $(63.2 \pm 29.3$ vs. $47.6 \pm 16.5 \mathrm{~s} ; p=0.029)$ and TMT-B (197.9 \pm 88.1 vs. $146.8 \pm 76.5 \mathrm{~s} ; p=0.03)$. No differences were observed with respect to age, gender, and education.
\end{abstract}

D. S. Torres $(\varangle) \cdot J$. Abrantes $\cdot$ C. E. Brandão-Mello

Federal University of the State of Rio de Janeiro (UNIRIO),

Rio de Janeiro, RJ, Brazil

e-mail: daniel.simplicio@hotmail.com

J. Abrantes

e-mail: jeffabrantes@uol.com.br

C. E. Brandão-Mello

e-mail: cedubrandao@gmail.com

D. S. Torres · J. Abrantes $\cdot$ C. E. Brandão-Mello Serviço de Gastroenterologia e Hepatologia do Hospital Universitário Gaffrée e Guinle (Hepatology Outpatient Clinic of the Gaffrée and Guinle University Hospital), Rua Mariz e Barros, 775, Tijuca,

Rio de Janeiro, RJ, Brazil 20270-004
In conclusion, MMSE proved to be a useful tool for detecting global cognitive impairment experienced by cirrhosis patients. Moreover, the most impaired cognitive functions were verbal episodic memory and information processing speed. These findings suggest that minimal hepatic encephalopathy represents a disorder that affects the medial temporal system and, possibly, the prefrontal cortex, and this requires further study.

Keywords Cirrhosis · Minimal hepatic encephalopathy · Cognitive tests $\cdot$ Cognitive impairment

\section{Introduction}

Since ancient Babylonian times (1894-1595 B.C.), people have been aware of the influence of liver dysfunction on cognition (Osler 2009). Today, cirrhosis is the seventh most common cause of death in the United States, with more than 25,000 deaths per year. In contrast, Brazil reports an average of 12,800 deaths per year due to cirrhosis, and it is estimated that approximately 3,500 new cases are identified annually (Lessa 1996).

Liver encephalopathy is a syndrome stemming primarily from cirrhosis, and is associated with a wide spectrum of neuropsychiatric and motor manifestations (Weissenborn et al. 2001b). Moreover, changes to a mental state can vary from subtle cognitive dysfunctions to coma. Motor changes can also be absent, or include variable degrees of tremor, asterixis, choreoathetotic movements, bradykinesia, hyporeflexia, and even pyramidal signals (Amodio et al. 2010). Within this spectrum, minimal liver encephalopathy is the mildest form of liver encephalopathy. It is characterized by subtle changes in cognitive function, neurophysiological parameters, neurotransmission, cerebral blood flow and liquid homeostasis, as well as an absence of observed motor disturbances or disruption of the sleep-wake cycle (Amodio et al. 2004).

To date, there is no gold standard for diagnosing the neuropsychiatric changes associated with minimal liver encephalopathy (Ferenci et al. 2003). However, based on the studies 
published to date, two observations can be made: (1) variables such as age, gender, educational level, and sociodemographic differences have a strong influence on a patient's performance (Amodio et al. 2004); and (2) the cutoff points used to define normal and abnormal performances are generally arbitrary, and moreover, do not consider distributions relevant to the population to which they are being applied (Córdoba 2011). Paradoxically, the majority of published studies do not account for the interference that can be caused by these factors.

Although cognitive disorders appear in the initial phases of cirrhosis and have a significant impact on a patient's quality of life, their prevalence is unknown. There is also no clear consensus regarding the sensitivity, specificity, and accuracy of the evaluation instruments available (Montagnese et al. 2004). Moreover, variables such as age, gender, educational levels, sociodemographic differences, psychiatric illnesses, the use of interferon, and clinical comorbidities can influence the results of cognitive evaluations, making them even more difficult to analyze (Valentine and Meyers 2005; Bajaj et al. 2010). Taken together, these factors indicate that further investigation is needed.

The primary objective of this study was to describe the cognitive changes that occurred in cirrhotic patients classified with Child-Turcotte-Pugh A and B that did not clinically manifest liver encephalopathy. The secondary objectives of this study were: (1) to describe the most common cognitive disturbances that occurred in patients with minimal liver encephalopathy; (2) to compare the cognitive performance of cirrhotic patients with controls paired according to age, gender, educational background, and sociodemographic characteristics, and (3) to identify the main psychometric tests that were altered by patients with minimal liver encephalopathy.

\section{Methodology}

Patients with cirrhosis aged 17 to 79 years were selected from the Hepatology Outpatient Clinic of the Medical Clinic of the Gaffrée and Guinle University Hospital, Rio de Janeiro, Brazil. These patients were selected according to one or more of the following inclusion criteria: (1) a liver biopsy confirmed cirrhosis based on METAVIR classification (Lefkowitch 2007); (2) an abdominal ultrasound detected reduction in the liver parenchyma and the presence of parenchymatous nodules, or (3) a high-resolution digestive endoscopy detected esophageal varices or portal hypertensive gastropathy.

In patients with cirrhosis, the following were considered to be exclusion criteria: less than 2 years of schooling, a Child-Pugh classification of $\mathrm{C}$, episodic or persistent liver encephalopathy at any time during clinical development, the use of interferon at any time during clinical development, the use of psychotropic drugs, the use of alcohol in the previous 6 months, use of illicit drugs in the previous
6 months, chronic obstructive pulmonary disease, neurological disease, psychiatric disease, neoplastic disease, thyroid disease, diabetes mellitus, cardiac insufficiency, renal insufficiency, adrenal insufficiency, visual deficiency, folic acid deficiency, vitamin B12 deficiency, being seropositive for syphilis or HIV, an infection within the previous 2 weeks, or digestive hemorrhage in the previous 2 weeks.

The control group consisted of healthy individuals which were selected to match with the patient group with respect to age ( \pm 3 years), gender, education ( \pm 1 year), and sociodemographic characteristics. Subjects were interviewed to exclude those with visual deficiency, less than 2 years of schooling, use of interferon, use of psychotropic medication, use of illicit drugs in the previous 6 months, chronic obstructive pulmonary disease, neurological disease, psychiatric illness, neoplastic disease, endocrine disease, cardiac insufficiency, renal insufficiency, malnourishment, syphilis, HIV, viral hepatitis, auto-immune hepatitis, or a history of heavy drinking. Minimal and moderate drinking were not exclusion criteria for the control group.

Before starting the evaluation, patients and controls were interviewed to identify each group's principal complaints. The questionnaire that was used addressed the following items: (1) difficulty in remembering things; (2) extent of dispersion or distraction; (3) reduction in reasoning speed; (4) difficulty in doing two things at the same time; (5) difficulty driving; and (6) other spontaneous neurological complaints.

Study instruments were selected according to the recommendations of the Scientific Department of Cognitive Neurology and Aging of the Brazilian Academy of Neurology (Nitrini et al. 2005). Participants were asked to respond to the Beck Depression Inventory (BDI) survey. In Brazil, scores between 0 and 11 were found to correspond with an absence of depression (Beck and Steer 2001). Therefore, individuals receiving a score of 12 or more were excluded and were sent for outpatient tracking. The remaining patients and controls completed the Mini-Mental State Examination (MMSE), a Simple Drawing Test, a Clock Drawing Test (Shulman's method), the Rey Auditory-Verbal Learning Test (RAVLT), a Random Letter Test, a Stroop Test (Dodrill's method), Trail-Making Test (TMT) A and B (Reitan's method), the Boston Naming Test, a Category Verbal Fluency Test, the Direct and Indirect Digit Span Test from the third edition of the Wechsler (2004) Adult Intelligence Scale (WAIS-III), the Constructional Praxis Test from the Consortium to Establish a Registry for Alzheimer's Disease (CERAD), a Processing Speed Test (WAIS-III), and a Similarities Test (WAIS-III). The average time to complete these tests was approximately $2 \mathrm{~h}$.

After the cognitive evaluation, blood was collected from each patient with cirrhosis to assay levels of thyroid stimulating hormone (TSH), free thyroxine (free T4), cortisol, folic acid, 
vitamin B12, and to perform blood tests for syphilis (VDRL) and human immunodeficiency virus (HIV). At this point of the research, the following were considered criteria for exclusion: $\mathrm{TSH}>5 \mu \mathrm{U} / \mathrm{ml}$, free $\mathrm{T} 4<0.8 \mathrm{ng} / \mathrm{dl}$, morning cortisol $<5 \mu \mathrm{g} / \mathrm{dl}$, folic acid $<5 \mathrm{ng} / \mathrm{ml}$, vitamin B12 $<250 \mathrm{pg} / \mathrm{ml}$, and seropositivity for syphilis or HIV. Hemoglobin levels $(\mathrm{Hb})$ and hematocrits (Hct) were also recorded to exclude cases of severe anemia (e.g., $\mathrm{Hb}<7 \mathrm{~g} / \mathrm{dl}$ or Hct $<21 \%$ ).

SPSS (v. 14, 2006) was used to analyze the data. To compare the performance of patients with cirrhosis to the control group, the non-parametric Mann-Whitney test (for more than two categorical variables) and Fisher's exact test (for two categorical variables) were applied. The chi-square test was used to compare proportions, such as gender and prevalence of cognitive complaints in the two groups. Spearman's rank correlation coefficient was also used to evaluate the relationship between age, educational background, and performance on cognitive tests. Descriptive levels with a $p$-value less than 0.05 were considered statistically significant.

\section{Results}

Initially, 184 patients with cirrhosis and 30 healthy individuals were evaluated between March 2010 and January 2011.

Of these cases, 155 cirrhosis patients were excluded. Figure 1 summarizes the number of exclusions in cirrhotic patients and controls in each stage of the research. Figure 2 shows the total distribution of the exclusion criteria in the cirrhosis group. Thirty patients were accepted for cognitive evaluation and, in a subsequent consultation, blood samples were obtained to determine levels of TSH, free T4, cortisol, folic acid, vitamin B12, VDRL, and HIV status. For one patient, blood level of vitamin B12 was $178 \mathrm{pg} / \mathrm{ml}$ (exclusion criteria: vitamin B12 $<250 \mathrm{pg} / \mathrm{ml}$ ). Therefore, this patient was excluded. Overall, 29 cases were selected for further analysis. The average hemoglobin level was 12.9 \pm $2.9 \mathrm{~g} / \mathrm{dl}$, and the average hematocrit was $39.3 \pm 7.9 \%$. The minimum values for hemoglobin and hematocrits were $10.6 \mathrm{~g} / \mathrm{dl}$ and $31.9 \%$, respectively, which indicated that the patient group did not have conditions of serious anemia.

Among healthy individuals, seven were excluded for being too young (by at least 3 years), or for completing more education (1 year or more) than the average level of the cirrhotic patients. One person was excluded for scoring higher than 11 points on the BDI. As a result, 22 people without cirrhosis participated as part of the control group.

Within the patient group, the principal etiological causes identified were chronic hepatitis C (72.4\%), hepatitis C associated with alcohol $(6.9 \%)$ and cryptogenic disease $(6.9 \%)$. The remaining etiological causes (e.g., alcohol, hepatitis B, hepatitis B associated with alcohol, and auto-immune hepatitis) represented a prevalence of $3.4 \%$ (Table 1).

The average age of the patients with cirrhosis was $59.8 \pm$ 8 years, while that of the controls was $58.1 \pm 9.1$ years. The average educational level was $8.5 \pm 3.9$ years and $9.1 \pm$
Fig. 1 Selection of cirrhotic patients and controls

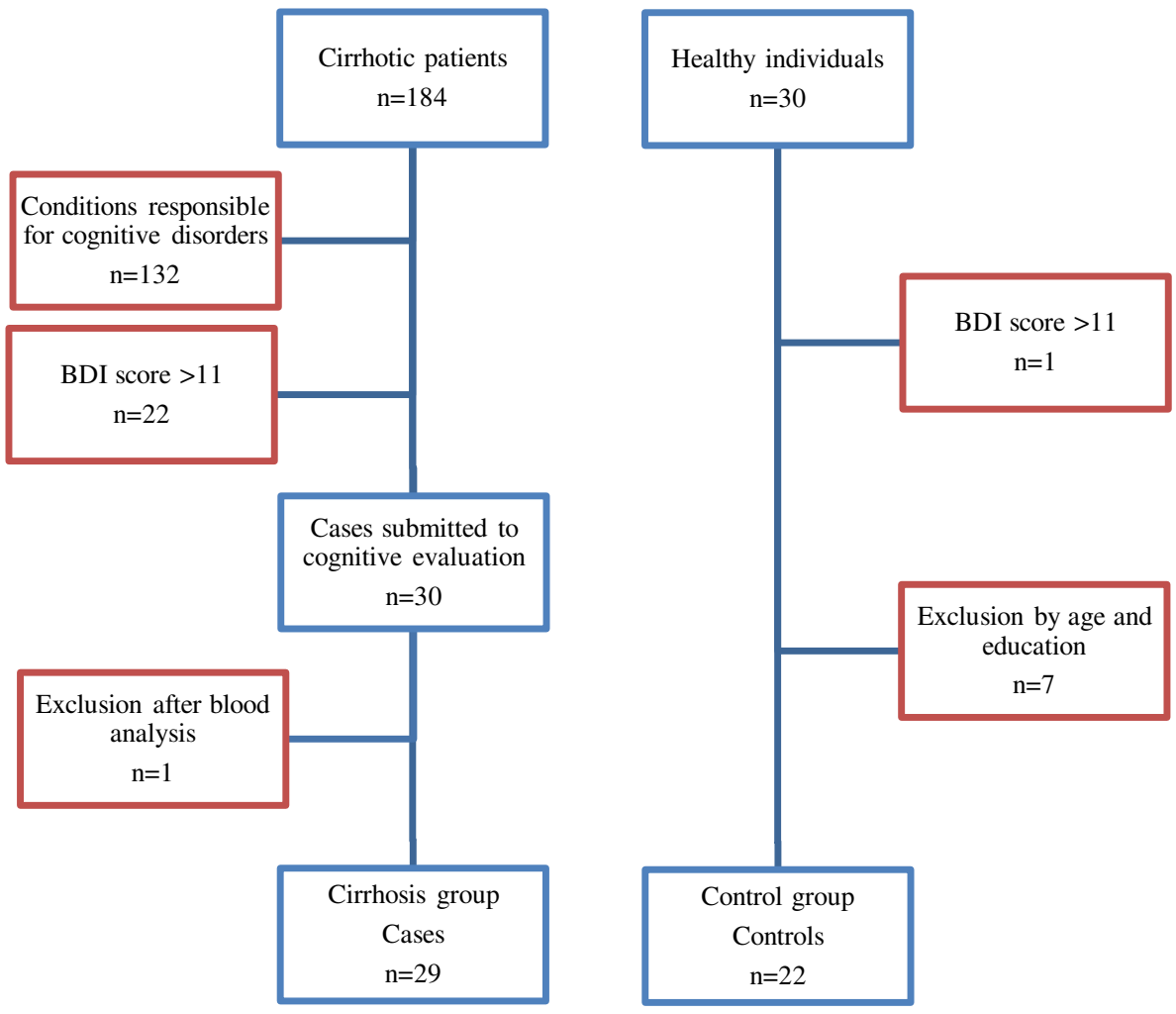


Fig. 2 Reasons for the exclusion of cirrhotic patients

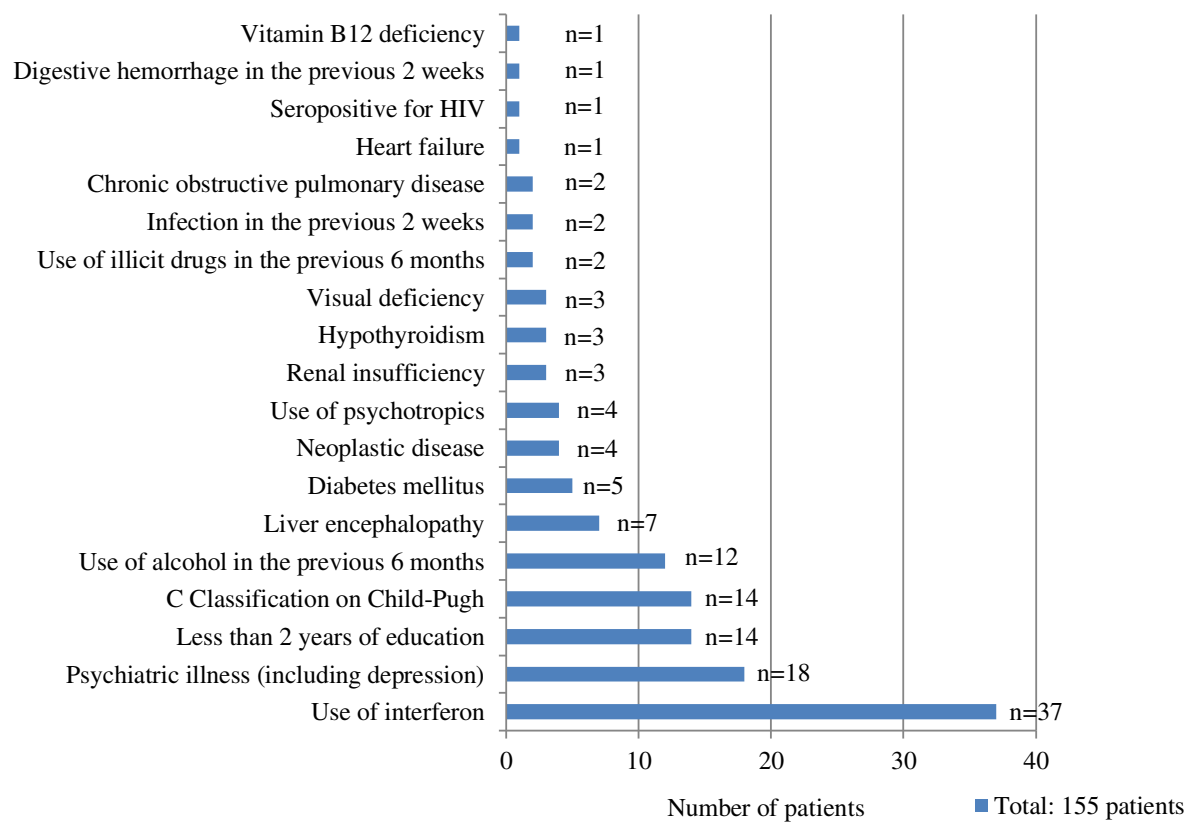

3 years, respectively. The Mann-Whitney test found no statistically significant difference between the groups with regard to age $(p=0.299)$ or educational level $(p=0.407)$.

The majority of the cohort $(69 \%)$ consisted of patients with an A5 Child-Pugh classification. For example, 23 (79.3\%) patients had a Child-Pugh classification of A, while only 6 (20.7\%) patients had a Child-Pugh classification of B. With respect to gender, $51.7 \%$ of the patients with cirrhosis were male, while $54.5 \%$ of the controls were male. Correspondingly, women constituted $48.3 \%$ and $45.5 \%$ of the two groups, respectively. The chi-square test did not verify

Table 1 Etiology and child-pugh classification of patients with cirrhosis

\begin{tabular}{lll}
\hline Etiology and Child-Pugh classification & \multicolumn{2}{l}{ Frequency } \\
\cline { 2 - 2 } & Absolute (n) & Relative (\%) \\
\hline Etiology & & \\
Alcoholic & 1 & $3.4 \%$ \\
Cryptogenic & 2 & $6.9 \%$ \\
Auto-immune hepatitis & 1 & $3.4 \%$ \\
Hepatitis B & 1 & $3.4 \%$ \\
Hepatitis B and alcoholic & 1 & $3.4 \%$ \\
Hepatitis C & 21 & $72.4 \%$ \\
Hepatitis C and alcoholic & 2 & $6.9 \%$ \\
Child-Pugh & & \\
5 & 20 & $69.0 \%$ \\
6 & 3 & $10.3 \%$ \\
7 & 5 & $17.2 \%$ \\
8 & 1 & $3.4 \%$ \\
\hline
\end{tabular}

a statistically significant difference between the cirrhosis patients and controls based on proportion of gender $(p=0.842)$.

The prevalence of cognitive complaints reported by cirrhotic patients versus the controls was evaluated using the chisquare test (Fig. 3), and no statistically significant difference in the symptoms of the two groups was detected $(p>0.05)$.

Overall, the prevalence of cognitive changes detected by psychometric tests in the cirrhosis group was $51.7 \%$. Regarding the evaluation of global cognitive performance, the control group had an average score of $28.1 \pm 1.8$ points on the MMSE (median, 28), while patients with cirrhosis had an average of $26.3 \pm 2$ points (median, 26) (Fig. 4). This difference was statistically significant $(p=0.004)$ (Table 2$)$.

When individual performances on each item of the MMSE were compared between the groups regarding temporal orientation, spatial orientation, immediate memory, attention and calculation, recall, language and visuoconstructive abilities, there was no significant difference $(p>0.05)$. However, cirrhotic patients exhibited a lower overall performance than the control group for these seven categories, particularly in attention and calculation.

On the RAVLT, the control group received an average learning score of $41 \pm 9.1$ points (median, 40.5), while the cirrhotic group received an average score of $35.4 \pm 9$ points (median, 34) (Fig. 5). This was a statistically significant difference $(p=0.041)$ (Table 2). For retrospective interference, the control group had an average score of $0.84 \pm 0.16$ (median, 0.88), and the cirrhotic group scored $0.67 \pm 0.22$ (median, 0.66) ( $p=0.004)$ (Fig. 6). Similarly, recognition was better preserved in the control group (mean, $11.2 \pm 4.1$ points; median, 11.5 points) versus the cirrhotic group (mean, $8.7 \pm$ 2.6 points; median, 9 points) ( $p=0.024)$ (Fig. 7). 
Fig. 3 Proportion of cognitive complaints reported by patients in the cirrhosis and control groups

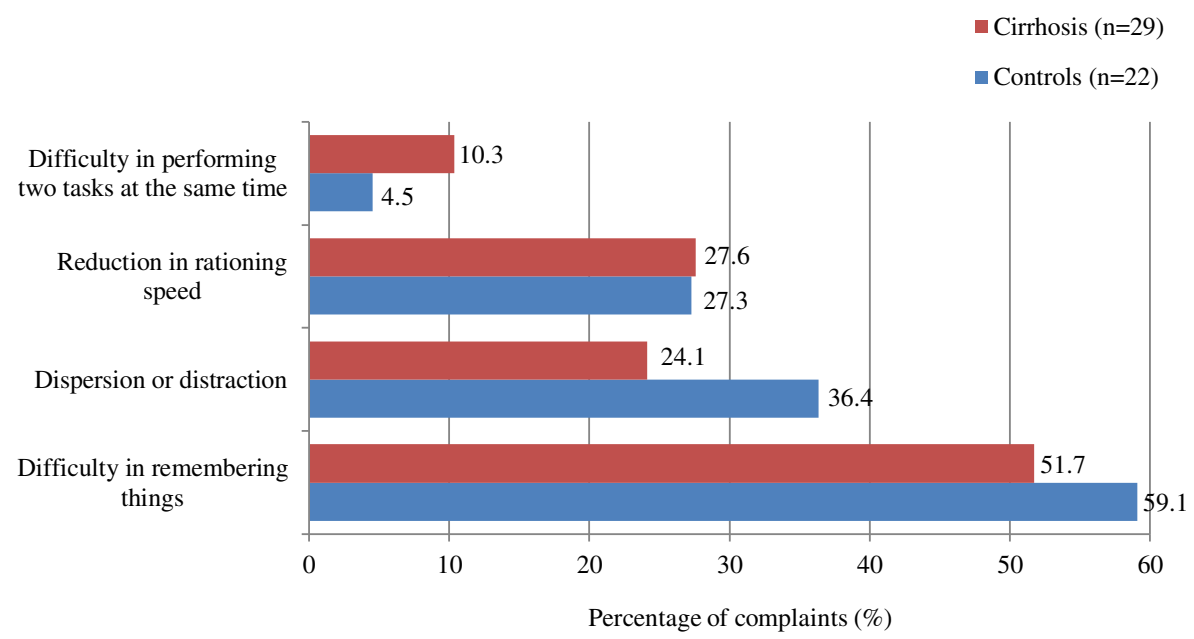

For the TMT-A, the control group needed an average of $47.6 \pm 16.5 \mathrm{~s}$ to complete the task, while the cirrhotic group needed $63.2 \pm 29.3 \mathrm{~s}$ (Table 2). However, as Fig. 8 shows, three controls and one patient exceeded the mean value. This can be observed according to the lowest averages achieved in both samples (41.5 $\mathrm{s}$ for the control group vs. $57 \mathrm{~s}$ for the cases). In addition, this difference was statistically significant $(p=0.029)$. For the TMT-B, the control group needed $146.8 \pm 76.5 \mathrm{~s}$ (median, $123.5 \mathrm{~s}$ ) to complete the task, while the cirrhotic group required $197.9 \pm 88.1 \mathrm{~s}$ (median, $192 \mathrm{~s}$ ) (Fig. 9). These differences also represented a statistically significant difference $(p=0.03)$ (Table 2$)$.

With respect to the other cognitive evaluation instruments used (e.g., Simple Drawing Test, Clock Drawing Test, Random Letter Test, Stroop Test, Boston Naming Test, Category Verbal Fluency Test, Direct and Indirect Digit Span Test, Constructional Praxis Test, Processing Speed Test, and Similarities Test), no statistically relevant

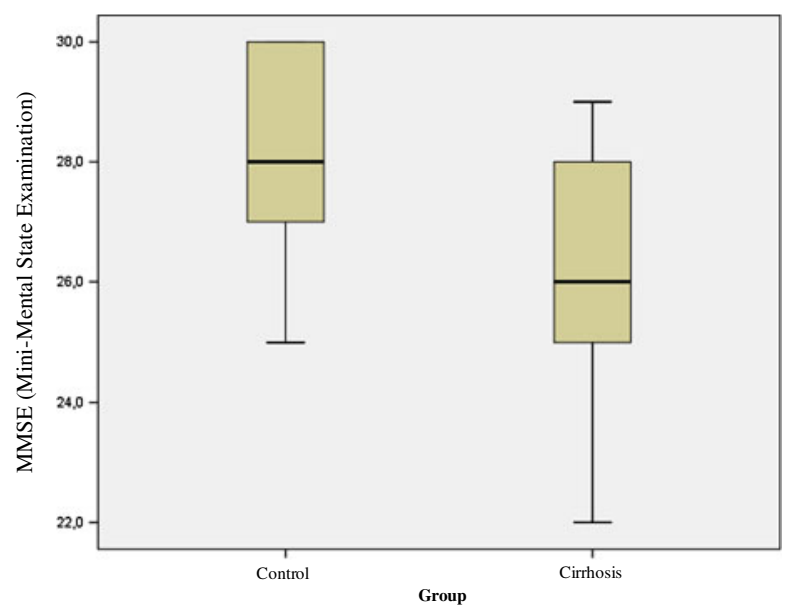

Fig. 4 Performance on mini-mental state examination by patients with cirrhosis versus the control group differences were observed between the cirrhotic group and the control group $(p>0.05)$ (Table 2).

Spearman's rank correlation coefficient was used to evaluate the relationship between the variables of age, educational level, and cognitive performance between the cirrhotic group and the control group. While age was not found to interfere with performance on any of the cognitive tests conducted, the educational level of the patients with cirrhosis was found to influence the total score for the MMSE, Boston Naming Test, Category Verbal Fluency Test, Constructional Praxis Test, Processing Speed Test, and Similarities Test. For the control group, the level of education affected learning on the RAVLT (the sum of A1 to A5) $(p=0.042)$. However, since the cirrhotic patients and controls were adequately paired, the educational levels of each group did not affect the overall results.

\section{Discussion}

The primary objective of this study was to describe cognitive disorders observed in the initial stages of cirrhosis (e.g., ChildPugh classification A and B). The secondary objectives were to describe the cognitive functions that were the most compromised, to compare the performance of cirrhotic patients with the performance of healthy individuals (controls), and to identify the standardized tests that detected these changes.

The initial group of cirrhotic patients identified exhibited a high prevalence of comorbidities. In addition, medications were found to be responsible for some cognitive changes observed in these patients. As a result, $84 \%$ of these preselected cases were later excluded.

This high exclusion rate was much greater than that described by previous studies. However, few publications had previously reported a screening for comorbidities that might cause associated cognitive disorders. In addition, none of the previous studies excluded patients who had used 
Table 2 Score Comparisons between Cirrhosis Patients versus the Control Group
MMSE mini-mental state examination, $S D T$ simple drawing test, $R A V L T$ Rey auditory-verbal learning test, $S D$ standard deviation

${ }^{a}$ Mann-Whitney test

\begin{tabular}{|c|c|c|c|c|c|c|c|}
\hline \multirow[t]{2}{*}{ Cognitive Test } & \multicolumn{3}{|c|}{ Controls $(n=22)$} & \multicolumn{3}{|c|}{ Cirrhosis $(n=29)$} & \multirow[t]{2}{*}{$p$-value ${ }^{\mathrm{a}}$} \\
\hline & Mean & SD & Median & Mean & SD & Median & \\
\hline MMSE & 28.1 & 1.8 & 28.0 & 26.3 & 2.0 & 26.0 & 0.004 \\
\hline Incidental memory (SDT) & 5.6 & 1.2 & 5.0 & 5.4 & 1.1 & 6.0 & 0.730 \\
\hline Immediate memory (SDT) & 8.5 & 0.7 & 8.0 & 8.2 & 1.1 & 8.0 & 0.296 \\
\hline Delayed visual memory (SDT) & 8.8 & 1.0 & 9.0 & 8.7 & 1.1 & 9.0 & 0.631 \\
\hline Clock drawing test & 4.06 & 0.8 & 5.0 & 4.1 & 1.3 & 5.0 & 0.128 \\
\hline Learning (RAVLT) & 41.0 & 9.1 & 40.5 & 35.4 & 9.0 & 34.0 & 0.041 \\
\hline Retroactive interference (RAVLT) & 0.84 & 0.16 & 0.88 & 0.67 & 0.22 & 0.66 & 0.004 \\
\hline Proactive interference (RAVLT) & 0.88 & 0.45 & 0.78 & 1.01 & 0.36 & 1.0 & 0.109 \\
\hline Forgetting speed (RAVLT) & 0.96 & 0.13 & 1.0 & 1.01 & 0.47 & 0.9 & 0.788 \\
\hline Recognition (RAVLT) & 11.2 & 4.1 & 11.5 & 8.7 & 2.6 & 9.0 & 0.024 \\
\hline Random letter test & 0.5 & 0.8 & 0 & 1.0 & 1.8 & 0 & 0.789 \\
\hline Stroop test (Part 1) & 116.9 & 19.0 & 112.5 & 127.0 & 46.1 & 120.0 & 0.327 \\
\hline Stroop test (Part 2) & 251.5 & 45.6 & 263.0 & 271.6 & 35.1 & 290.0 & 0.105 \\
\hline Stroop test (Part 2 - Part 1) & 139.1 & 48.0 & 142.5 & 144.6 & 39.5 & 149.0 & 0.575 \\
\hline TMT-A & 47.6 & 16.5 & 41.5 & 63.2 & 29.3 & 57.0 & 0.029 \\
\hline TMT-B & 146.8 & 76.5 & 123.5 & 197.9 & 88.1 & 192.0 & 0.030 \\
\hline TMT (B/A) & 3.1 & 1.45 & 2.76 & 3.32 & 1.47 & 3.0 & 0.441 \\
\hline Boston naming test & 14.0 & 1.1 & 14.0 & 13.9 & 1.4 & 14.0 & 0.693 \\
\hline Verbal fluency test & 18.7 & 5.0 & 17.5 & 15.8 & 4.6 & 16.0 & 0.053 \\
\hline Direct digit span test & 7.7 & 1.9 & 7.5 & 8.1 & 1.8 & 8.0 & 0.445 \\
\hline Indirect digit span test & 4.1 & 1.9 & 4.0 & 4.1 & 1.8 & 4.0 & 0.698 \\
\hline Constructional praxis test & 1.0 & 0.9 & 1.0 & 1.2 & 1.0 & 1.0 & 0.334 \\
\hline Processing speed test & 22.1 & 8.6 & 23.0 & 17.4 & 9.2 & 16.0 & 0.072 \\
\hline Similarities test & 18.5 & 6.6 & 18.0 & 15.1 & 7.3 & 16.0 & 0.090 \\
\hline
\end{tabular}

Of the patients admitted after the initial evaluation, 22 $(43.2 \%)$ were excluded because they scored more than 11 points on the BDI. It is possible that this large number of exclusions corresponds to false positive results, particularly since the BDI considers bodily complaints such as insomnia, fatigue, loss of appetite, loss of weight, a preoccupation with abdominal pain, and loss of libido in formulating a score. Moreover, these symptoms can occur during cirrhosis and yet not be associated with depression. However, in the present study, BDI scores were used for selection since little emphasis is given in clinical practice to emotional conditions and social attitudes in the initial stages of cirrhosis (Bianchi et al. 2005).

Mood disturbances are as prevalent as the cognitive changes that accompany minimal liver encephalopathy (Miotto et al. 2010). Therefore, the relationship between the seriousness of cirrhosis and BDI scores for those excluded were investigated. There was no statistically significant correlation found between the seriousness of a cirrhosis condition and the presence of depression, but these results may be due to the small sample size analyzed. In addition, the role of the serotoninergic system in regulating mood is well-known (Stahl 2008), and its function is affected by hyperammonemia and is sensitive to the port-system shunt grade (García- 


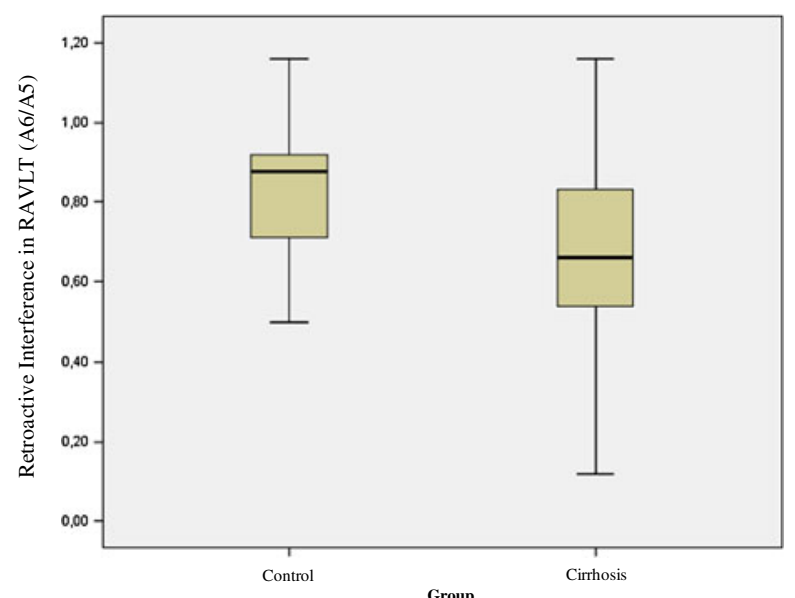

Fig. 6 Retroactive interference for patients with cirrhosis versus the control group

Martinez and Córdoba 2011). Thus, the hypothesis that a greater prevalence of mood disorders in cirrhotic patients is related to the cirrhosis condition should not be discarded.

The final cohort consisted of 29 cirrhotic patients, and these patients did not experience serious anemia based on the average and minimum values for hemoglobin and hematocrits recorded. Anemia could represent a potentially confusing factor for poor performance, therefore, it was important that this condition be excluded. For patients who reported drinking, or drinking in combination with having an autoimmune hepatitis condition, their scores were higher than those of the other non-drinking cirrhosis patients in nearly all of the evaluation instruments applied. The exceptions to this observation were the scores for "immediate memory" on the Simple Drawing Test, "recognition" on the RAVLT and Direct Digit Span Test. The subgroup of alcoholic patients with or without autoimmune hepatitis were also younger in age and had completed higher levels

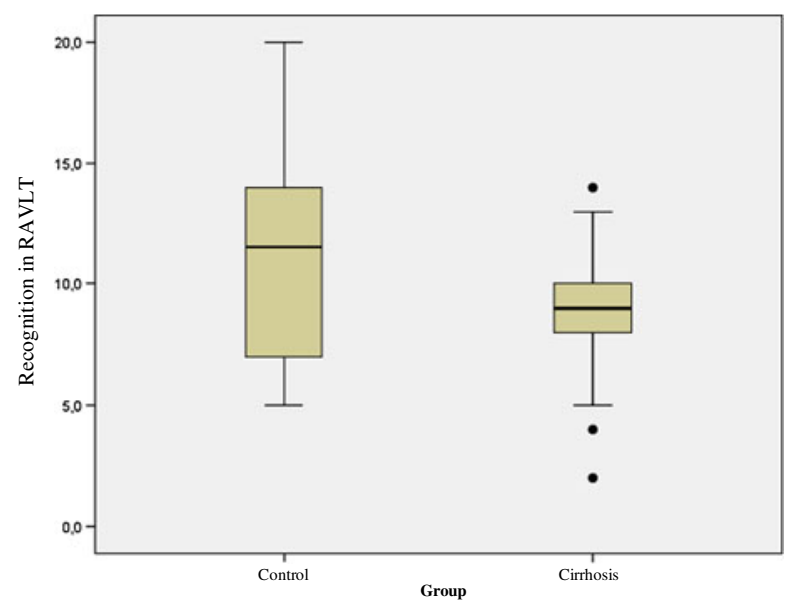

Fig. 7 Recognition by cirrhosis patients versus the control group

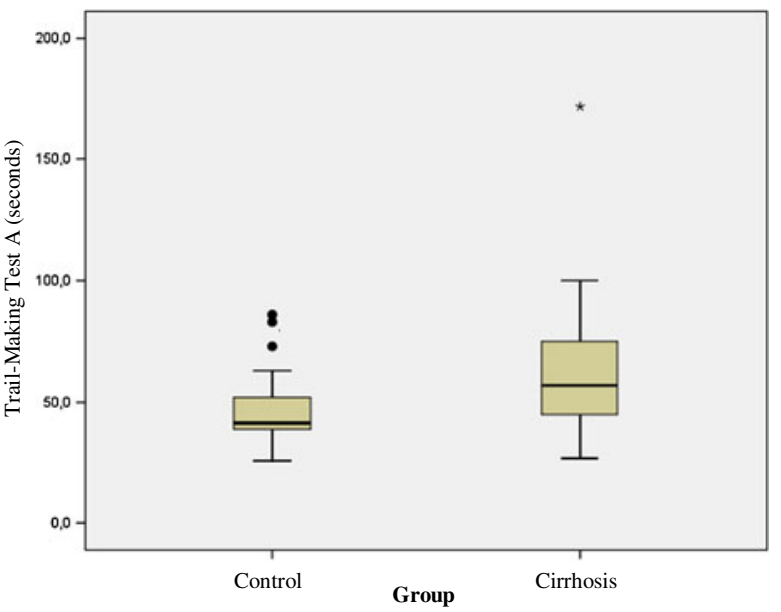

Fig. 8 Results of trail-making test a for cirrhosis patients versus the control group

of education than the other cirrhotic cases, and this may have improved their performance. It was also observed that the etiology of cirrhosis did not produce clinically relevant or statistically significant differences, and this is consistent with the literature (Amodio et al. 2005, 2010).

The main symptoms reported by cirrhosis patients included "difficulties in remembering things" (59.1\%), "dispersion or distraction" (36.4\%), "reduction in the speed of reasoning" (27.4\%), and "difficulty in performing two tasks at the same time" (4.5\%). There were no reports of "difficulty driving", although only five patients had national habilitation licenses.

There was also no statistically significant difference in the prevalence of cognitive complaints reported by the cirrhotic group versus the healthy controls. Figure 3 shows that symptoms such as "difficulty remembering things" and "dispersion and distraction" were proportionally more common in the control group. Therefore, taken together, these results appear to indicate that in the initial stages of cirrhosis, spontaneous

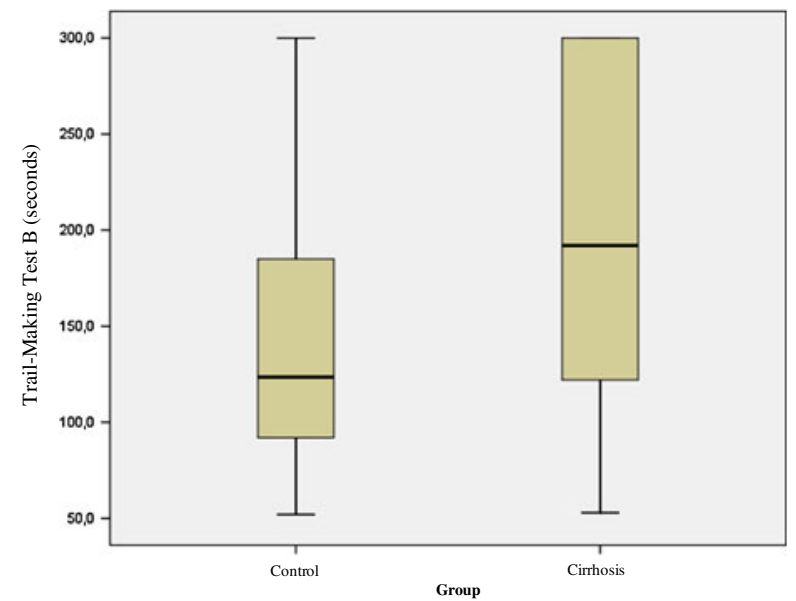

Fig. 9 Results of trail-making test B for cirrhosis patients versus the control group 
reporting is not a reliable parameter for detecting the presence of cognitive deficits.

The main psychometric tests affected by minimal liver encephalopathy were the MMSE, RAVLT, and TMT-A and B. The MMSE score, which evaluates global cognitive performance, proved to be a useful instrument for tracking minimal liver encephalopathy $(p=0.004)$. This finding is consistent with work by Kramer et al. (2002) which evaluated the performance of 22 patients with Child-Pugh B and $\mathrm{C}$ cirrhosis who had received a transjugular intra-hepatic port-systemic shunt (TIPS) and were compared to 22 healthy controls. The performance of Kramer's patients was slightly higher than that found in this study $(28.8 \pm 1.3$ points vs. $28.1 \pm 1.8$ points, respectively), which may be due to differences in age, education, and sociodemographic factors between the two cohorts analyzed.

Since few studies have examined the applicability of MMSE in screening for liver encephalopathy, it was an important finding in the present study that patients with cirrhosis received an average score of $26.3 \pm 2$ points (median, 26) on the MMSE, while the control group had an average score of $28.1 \pm 1.8$ points (median, 28). Once this assessment can be easily applied and widely used (Brucki et al. 2003), more studies are needed to determine its sensitivity and specificity in screening for minimal hepatic encephalopathy, as this condition is often underdiagnosed (Ferenci et al. 2003).

In general, patients with cirrhosis had a lower performance than controls in the seven categories of the MMSE (e.g., temporal orientation, spatial orientation, immediate memory, attention and calculation, recall, language, and visuoconstructive abilities). Unfortunately, the small sample size of the groups analyzed prevented a statistical evaluation of which MMSE items were the most affected.

Previously, studies that made a distinction between visual and verbal memory reported similar rates of compromise (Bahceci et al. 2005). Deficits in visual perception, incidental visual memory, immediate visual memory, and delayed visual memory were evaluated using the Simple Drawing Test (Nitrini et al. 2004). This instrument evaluates both visual recall (based on shapes that needed to be identified and named) and the ability to integrate verbal representation. Alternatively, the RAVLT was used to evaluate verbal memory. For cirrhotic patients, a deficit in learning on the RAVLT (e.g., the sum of A1 to A5) was associated with the initial stages of disease, and suggested that a compromise in this modality of storing information was present. Furthermore, the difference in learning scores between the cirrhotic group and the control group were statistically significant $(p=0.041)$. These findings are consistent with those of Ortiz et al. (2006) who evaluated the performance of 99 patients with cirrhosis (Child-Pugh classification A, B, and C) that did not exhibit a clinical manifestation of liver encephalopathy, compared with 75 healthy controls.
When Spearman's correlation was used to evaluate the relationship between learning and recognition in patients who completed the RAVLT, higher scores from the sum of A1 to A5 were associated with better performance on the task of recognition ( $p=0.005$; Spearman's correlation coefficient $=0.509$ ). Thus, the changes observed in cirrhosis patients appear to indicate a dysfunction in memory storage has occurred. Squire and Zola-Morgan (1991) improved the structural memory model originally developed by Milner and Scoville in 1957. This model involves the medial temporal lobe, which is composed of the hippocampal formation (including the dentate gyrus, hippocampus, subiculum complex and entorhinal cortex), the perirhinal cortex, and the parahippocampal cortex. The latter two regions, anatomically situated adjacent to the entorhinal cortex, not only serve as a means of connecting the neocortex to the hippocampus, but also act as important sites for memory storage. Thus, deficits encountered on the RAVLT not only suggest a hippocampal dysfunction, but rather dysfunction that further affects the entire medial temporal system. Furthermore, compromise of the medial temporal system can be multimodal, that is, independent of the sensory modes through which information is presented (Squire et al. 2004). Compared with the Simple Drawing Test, compromise of learning according to the RAVLT suggests that verbal memory disturbances are more apparent in the initial stages of cirrhosis, and this is consistent with the results of the study.

On the RAVLT, answers for A6/A5 address retroactive interference. Using this instrument, the ability of an individual to evaluate words in an original list after the same words are combined with an interference list of words is compared. This is intended to detect whether a loss of new memory traces has occurred, or to what extent the storage of new information impedes the recall of longer-held information (Baddeley et al. 2009). In the present study, patients with cirrhosis exhibited a statistically significant difference in regard to this capacity compared to controls $(p=0.004)$.

In addition to recall, recognition was also compromised in patients with minimal liver encephalopathy. For example, when patients were asked to identify from an extensive list of words those which had been previously presented, their mean performance was worse than that of the controls $(8.7 \pm$ 2.6 points vs. $11.2 \pm 4.1$ points, respectively). This data suggests not only a encoding/storage problem, but also a retrieval deficit, and possibly outlines a disturbance in executive control process (Kim and Cabeza 2009). Since the prefrontal cortex supports strategic recall and plays a critical role in episodic encoding and retrieval process for both verbal and non-verbal material (Gagnon et al. 2010), its involvement in minimal hepatic encephalopathy requires further study.

It has previously been shown that an evaluation of attention in patients with minimal liver encephalopathy is key, as 
all other cognitive functions depend on proper functioning of attention (Amodio et al. 2005). Attention can be divided into three categories: sustained attention (vigilance), selective attention (inhibitory control), and divided attention (the ability to execute multiple tasks simultaneously).

Deficits in sustained attention are indicators of a compromise of the function of the right dorsolateral pre-frontal and left parietal cortex, a reduction in the activity of the ascending reticular activation system, or a decrease in corticoreticular-cortical influences (Weissenborn et al. 2005). Sustained attention was evaluated using the Random Letter Test, and no statistically significant difference was found between patients with cirrhosis and healthy individuals.

Selective attention, in turn, is only possible when, in addition to vigilance, there exists the ability to select sensory information and to inhibit distracting stimuli. Moreover, for selective attention to function properly, it needs the activity of the dorsolateral pre-frontal cortex, the parietal cortex, the frontal occulomotor fields, the superior colliculus, and the pulvinar of the thalamus (Weissenborn et al. 2005). Weissenborn et al. (2001a) and Amodio et al. (2005, 2010) previously described deficits in selective attention in cirrhotic patients, although no mention was made whether patients treated with interferon had been excluded. In the present study, we found no statistically significant difference between cirrhotic patients and the controls.

Finally, divided attention corresponds to the highest level of attention, and contributes to executive functions. Executive functions are important for the simultaneous awareness of context, options, consequences, relevance and emotional impact that allows the formulation of adaptative inferences, decisions, and actions (Longo et al. 2012). While the neuroanatomical basis for this system remains unclear, it is hypothesized that its ability to function is dependent on an integration of the anterior portion of the gyrus cinguli, the supplementary motor area, the orbitofrontal cortex, the dorsolateral prefrontal cortex, the thalamus, and the basal nuclei (Weissenborn et al. 2005).

TMT-A and B were used to evaluate divided attention. It was observed that patients with cirrhosis took more time to complete the TMT-A than the controls $(p=0.029)$, and discrepancies in performance were even greater for the TMT-B $(p=0.03)$. However, it should be emphasized that both groups had a higher performance time than the usual cutoff of $120 \mathrm{~s}$.

One possible reason for the prolonged time needed to execute the TMT-A, which requires less divided attention activity and cognitive flexibility, is a decrease in the speed of information processing. Curiously, patients with cirrhosis didn't have a statistically inferior performance compared to the control group on the Processing Speed Test. This result may be due to lower sensitivity associated with the Processing Speed Test in relation to the TMT, or to the small sample size.
Information processing speed can be affected by imbalances in neurotransmission, structural disorganization of the neural systems, deficiency of prefrontal cortex organization and direction of information flow, or demyelination (Rao 1995). Correspondingly, the first three mechanisms have previously been associated with the physiopathology of liver encephalopathy (Weissenborn et al. 2005; García-Ayllón et al. 2008; García-Martinez and Córdoba 2011).

A major obstacle in the interpretation of the TMT-B is the strong influence of education (Tombaugh 2004). For example, when Bragagnolo et al. (2009) studied the applicability of the TMT-B for 48 Brazilian patients with cirrhosis, they found that populations with low, or very low, levels of education needed more time to execute the task. For this reason, its use in clinical practice is limited due to an absence of well-established cutoff points. Moreover, in addition to the influence of education, performance on the $\mathrm{B}$ form highly correlates with performance on the A form, and is only moderately sensitive to changes in cognitive flexibility (Lezak et al. 2004). In this study, both patients with cirrhosis and the controls had mean $\mathrm{B} / \mathrm{A}$ reasoning scores greater than three. Although the median for the controls was below this limit (e.g., 2.76), due to the more symmetrical distribution of the data, there was no statistically significant difference in the $\mathrm{B} / \mathrm{A}$ indices for the two groups (Table 2). This finding suggests that a decrease in information processing speed was responsible for the delay in executing TMT-A and B by the cirrhosis patients, and this is consistent with the literature (Randolph et al. 2009; Kappus and Bajaj 2012).

Executive function, divided attention, and working memory are closely related. In the latter case, working memory corresponds to the temporary ability of an individual to reflect on, process, and store information in a short duration while engaged with other tasks. The aim of the Indirect Digit Span Test is to evaluate this function, which is related to the activity of the left fronto-parietal cortex and the gyrus cinguli (Weissenborn et al. 2005). In the present study, no statistically significant difference was found between cirrhotic patients and the controls on the Indirect Digit Span Test. In fact, patients with minimal liver encephalopathy exhibited average and median scores, as well as standard deviations, that were very similar to those of healthy individuals (Table 2).

In analyzing the other components of executive function, deficits in fluency (e.g., the ability to emit behaviors according to explicit or implicit rules) were not observed. In addition, the Verbal Fluency Test did not detect any statistically significant differences in verbal performance for the patients with cirrhosis versus the controls. In fact, the majority of patients were able to name more than 12 animals in $1 \mathrm{~min}$. Similarly, the Clock Drawing Test did 
not detect any statistically relevant differences in nonverbal fluency between the two groups. Finally, the Similarities Test did not identify differences between the abilities of the two groups to categorize and generate abstractions. Therefore, language proved to be strongly preserved in patients with minimal liver encephalopathy, and statistically relevant differences were not detected in the Boston Naming Test. In addition, there was no difference in the visuoconstructive abilities of the cirrhosis group versus the control group that were evaluated using the Constructional Praxis Test, with both groups making most of their mistakes in copying rhomboids and cubes.

A major obstacle in evaluating memory is the compromise of attention. Since an adequate performance regarding the former depends on the latter, attention is essential for learning (Amodio et al. 2005). Moreover, the memory and attention systems share common anatomical elements, despite employing different neurotransmitters (Weissenborn et al. 2005). The application of Spearman's correlation demonstrated that in cirrhotic patients, lower execution times for the TMT-B were associated with higher scores on the RAVLT. Moreover, attention capacity was found to be relatively well-preserved for patients with minimal liver encephalopathy. Therefore, taken together, these results suggest that compromise of the memory storage system is a key issue for cirrhosis patients.

Some drawbacks of the research can be pointed out: (1) the small number of participants, due to the rigidity of the exclusion criteria, (2) the fact that the control group did not undergo a blood sample analysis as the cirrhosis group, and (3) the study was not conducted in a blinded way. On the other hand, its positive points were: (1) it matched patients with cirrhosis with healthy controls on critical variables that can independently impact test performance, including age and level of education, (2) it used a number of well validated cognitive tests that are widely employed by neuropsychologists, and (3) the battery of tests was larger and more diverse in composition than is normally employed in literature, which enabled to consider the potential effects of minimal hepatic encephalopathy across a variety of cognitive domains.

In conclusion, the results of this study verify the most affected tests were MMSE, RAVLT, TMT-A and B. MMSE proved to be a useful tool for detecting global cognitive impairment experienced by cirrhosis patients. Patients with minimal liver encephalopathy tend to manifest difficulties in verbal episodic memory and in the speed of information processing. Despite this, other cognitive abilities remain relatively well-preserved. Taken together, these findings suggest that minimal liver encephalopathy is a disorder that compromises the medial temporal system and, possibly, the prefrontal cortex, and this requires further study.
Acknowledgments The authors would like to thank Carl B. Dodrill, $\mathrm{PhD}$, for providing the rules for application and for allowing the translation of the Stroop Test into Portuguese.

Open Access This article is distributed under the terms of the Creative Commons Attribution License which permits any use, distribution, and reproduction in any medium, provided the original author(s) and the source are credited.

\section{References}

Amodio P, Montagnese S, Gatta A, Morgan MY (2004) Characteristics of minimal hepatic encephalopathy. Metab Brain Dis 19:253367

Amodio P, Schiff S, Piccolo FD, Mapelli D, Gatta A, Umiltà C (2005) Attention dysfunction in cirrhotic patients: an inquiry on the role of executive control, attention, orienting and focusing. Metab Brain Dis 20:115-127

Amodio P, Ridola L, Schiff S, Montagnese S, Pasquale C, Nardelli S, Pentassuglio I, Trezza M, Marzano C, Flaiban C, Angeli P, Cona G, Bisiacchi P, Gatta A, Riggio O (2010) Improving the inhibitory control task to detect minimal hepatic encephalopathy. Gastroenterology 139:510-518

Baddeley A, Anderson MC, Eysenck MW (2009) Memory. Psychology Press, Hove, pp 198-214

Bahceci F, Yldirim B, Karincaoglu M, Dogan I, Spahi B (2005) Memory impairment in patients with cirrhosis. J Natl Med Assoc 97:213-216

Bajaj JS, Schubert CM, Heuman DM, Wade JB, Gibson DP, Topaz A, Saeian K, Hafeezullah M, Bell DE, Sterling RK, Stravitz RT, Luketic V, White MB, Sanyal AJ (2010) Persistence of cognitive impairment after resolution of overt encephalopathy. Gastroenterology 138:23322340

Beck AT, Steer RA (2001) Manual da versão em português das escalas Beck. Cunha JA. Casa do Psicólogo, São Paulo, pp 11-13

Bianchi G, Marchesini G, Nicolino F, Graziani R, Sgarbi D, Loguercio C, Abbiati R, Zoli M (2005) Psychological status and depression in patients with liver cirrhosis. Dig Liver Dis 37:593-600

Bragagnolo MA Jr, Teodoro V, Lucchesi LM, Ribeiro TCR, Tufik S, Kondo M (2009) Minimal hepatic encephalopathy detection by neuropsychological and neurophysiological methods and the role of ammonia for its diagnosis. Arq Gastroenterol 46:43-49

Brucki SMD, Nitrini R, Caramelli P, Bertolucci PHF, Okamoto IVH (2003) Suggestions for utilization of the mini-mental state examination in Brazil. Arq Neuropsiquiatr 61:777-781

Córdoba J (2011) New assessment of hepatic encephalopathy. J Hepatol 54:1030-1040

Ferenci P, Lockwood A, Mullen K, Tarter R, Weissenborn K, Blei AT, Members of the Working Party (2003) Hepatic encephalopathy - definition, nomenclature, diagnosis, and quantification: final report of the working party at the 11th World Congresses of Gastroenterology, Vienna, 1998. Hepatology 35:716-721

Gagnon G, Blanchet S, Grondin S, Schneider C (2010) Paired-pulse transcranial magnetic stimulation over the dorsolateral prefrontal cortex interferes with episodic encoding and retrieval for both verbal and non-verbal materials. Brain Res 1344:148-158

García-Ayllón MS, Cauli O, Sylveira MX, Rodrigo R, Candela A, Compañ A, Jover R, Pérez-Mateo M, Martínez S, Felipo V, Sáez-Valero J (2008) Brain cholinergic impairment in liver failure. Brain 131:2946-2956

García-Martinez R, Córdoba J (2011) Acute-on-chronic liver failure: the brain. Curr Opin Crit Care 17:177-183 
Kappus MR, Bajaj JS (2012) Covert hepatic encephalopathy: not as minimal as you might think. Clin Gastroenterol Hepatol 10:1208-1219

Kim H, Cabeza R (2009) Common and specific brain regions in highversus low-confidence recognition memory. Brain Res 1282:103-113

Kramer L, Bauer E, Gendo A, Funk G, Madl C, Pidlich J, Gangl A (2002) Neurophysiological evidence of cognitive impairment in patients without hepatic encephalopathy after transjugular intrahepatic portosystemic shunts. Am J Gastroenterol 97:162-166

Lefkowitch JH (2007) Liver biopsy assessment in chronic hepatitis. Arch Med Res 38:634-643

Lessa I (1996) Liver cirrhosis in Brazil: mortality and productive years of life lost prematurely. Bol Oficina Sanit Panam 121:111-122

Lezak MD, Howieson DB, Loring DW (2004) Neuropsychological assessment. Oxford University, New York, pp 368-374

Longo DL, Fauci AS, Kasper DL, Hauser SL, Jameson JL, Loscalzo J (2012) Harrison's principles of internal medicine. McGraw-Hill, New York, pp 210-211

Miotto EC, Campanholo KR, Machado MAR, Benute GGR, Lucia MCS, Fráquas R Jr, Bacchella T, Machado MCC (2010) Cognitive performance and mood in patients on the waiting list for liver transplantation and their relation to the model for endstage liver disease. Arq Neuropsiquiatr 68:62-66

Montagnese S, Amodio P, Morgan MY (2004) Methods for diagnosing hepatic encephalopathy in patients with cirrhosis: a multidimensional approach. Metab Brain Dis 19:281-312

Myint AM, Schwarz MJ, Steinbusch HWM, Leonard BE (2009) Neuropsychiatric disorders related to interferon and interleukins treatment. Metab Brain Dis 24:55-68

Nitrini R, Caramelli P, Herrera Júnior E, Porto CS, Charchat-Fichman H, Carthery MT, Takada LT, Lima EP (2004) Performance of illiterate and literate nondemented elderly subjects in two tests of long-term memory. J Int Neuropsychol Soc 10:634-638

Nitrini R, Caramelli P, Bottino CMC, Damasceno BP, Brucki SMD, Anghinah R (2005) Diagnosis of Alzheimer's disease in Brazil: cognitive and functional evaluation. Recommendations of the Scientific Department of Cognitive Neurology and Aging of the
Brazilian Academy of Neurology. Arq Neuropsiquiatr 63:720 727

Ortiz M, Córdoba J, Jacas C, Flavià M, Esteban R, Guardia J (2006) Neuropsychological abnormalities in cirrhosis include learning impairment. J Hepatol 44:104-110

Osler W (2009) The evolution of modern medicine. Echo Library, Fairford, pp 20-26

Randolph C, Hilsabeck R, Kato A, Kharbanda P, Li YY, Mapelli D, Ravdin LD, Romero-Gomez M, Stracciari A, Weissenborn K, International Society for Hepatic Encephalopathy and Nitrogen Metabolism (2009) Neuropsychological assessment of hepatic encephalopathy: ISHEN practice guidelines. Liver Int 29:629-635

Rao SM (1995) Neuropsychology of multiple sclerosis. Curr Opin Neurol 8:216-220

Squire LR, Zola-Morgan S (1991) The medial temporal lobe memory system. Science 253:1380-1386

Squire LR, Stark CEL, Clark RE (2004) The medial temporal lobe. Annu Rev Neurosci 27:279-306

Stahl SM (2008) Stahl's essential psychopharmacology - neuroscientific basis and practical applications. Syndicate of the Press of The University of Cambridge, Cambridge, pp 195-222

Tombaugh TN (2004) Trail making test A and B: normative data stratified by age and education. Arch Clin Neuropsychol 19:203-214

Valentine AD, Meyers CA (2005) Neurobehavioral effects of interferon therapy. Curr Psychiatry Rep 7:391-395

Wechsler D (2004) Escala de Inteligência Wechsler para Adultos. Silva MCVM. Casa do Psicólogo, São Paulo

Weissenborn K, Heidenreich S, Ennen J, Rückert N, Hecker H (2001a) Attention deficits in minimal hepatic encephalopathy. Metab Brain Dis 16:13-19

Weissenborn K, Ennen J, Schomerus H, Rückert N, Hecker H (2001b) Neuropsychological characterization of hepatic encephalopathy. $\mathrm{J}$ Hepatol 34:768-773

Weissenborn K, Giewekemeyer K, Heidenreich S, Bokemeyer M, Berding G, Ahl B (2005) Attention, memory and cognitive function in hepatic encephalopathy. Metab Brain Dis 20:359-367 